\title{
Scènes et repas
}

La performance du repas et de la mort chez Rodrigo García et Vassilis Ziogas

Scenes of Meal: The Performance of Food in Rodrigo Garcia and Vassilis Ziogas

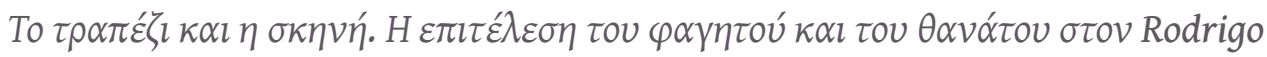

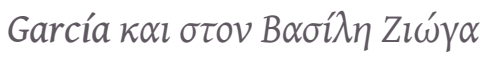

\section{Georges P. Pefanis}

\section{(Q) OpenEdition}

Journals

Édition électronique

URL : https://journals.openedition.org/ceb/6123

DOI : $10.4000 /$ ceb.6123

ISSN : 2261-4184

Éditeur

INALCO

Édition imprimée

ISBN : 978-2-85831-230-6

ISSN : 0290-7402

Référence électronique

Georges P. Pefanis, «Scènes et repas », Cahiers balkaniques [En ligne], Hors-série | 2016, mis en ligne le 16 mars 2017, consulté le 06 juillet 2021. URL : http://journals.openedition.org/ceb/6123 ; DOI : https://doi.org/10.4000/ceb.6123

Ce document a été généré automatiquement le 6 juillet 2021.

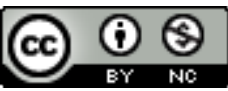

Cahiers balkaniques est mis à disposition selon les termes de la Licence Creative Commons Attribution - Pas d'Utilisation Commerciale 4.0 International. 


\title{
Scènes et repas
}

La performance du repas et de la mort chez Rodrigo García et Vassilis Ziogas

\author{
Scenes of Meal: The Performance of Food in Rodrigo Garcia and Vassilis Ziogas

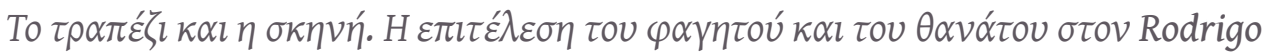

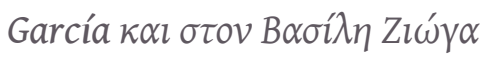

Georges P. Pefanis

Dans cet essai je voudrais rapprocher certaines pièces de Rodrigo García et Vassilis Ziogas, afin d'examiner la possibilité d'un passage caché entre $\beta p \omega ́ \sigma n \varsigma$ (manger) et

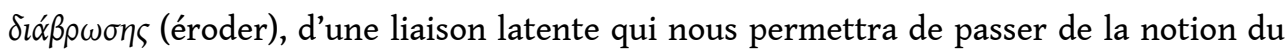

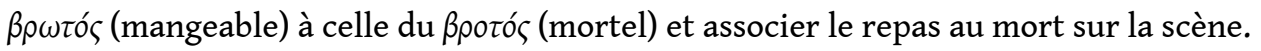

2 Nous devons accepter d'abord avec Jean-Pierre Poulain le fait que «l'aliment n'est pas un produit de consommation banal, il s'incorpore. Il entre dans le corps du mangeur, devient le mangeur lui-même, participant physiquement et symboliquement au maintien de son intégrité et à la construction de son identité » $(2013,39)$. Dans le cas du repas, l'incorporation est prise au pied de la lettre. Ensuite, et c'est le point crucial de notre approche, tandis que manger est une action humaine fondamentale, inextricablement entrelacée avec la vie, elle est en même temps un jeu avec la mort au fur et à mesure que la vie et la santé du sujet mangeur sont en jeu chaque fois que la décision d'incorporation est prise (FISCHLER, 2001, 69).

\section{Les scènes de Rodrigo García}

Dans le théâtre de Rodrigo García, la nourriture et la boisson deviennent le champ privilégié de la critique déconstructive des démocraties occidentales. Même si luimême prétend ne pas faire de politique, mais du théâtre, même s'il se fixe comme principal objectif d'inventer des formes et des formats sur la scène (TACKELS, 2009, 90), l'alimentation, qui constitue la base de son langage scénique dans beaucoup de ses œuvres, est à elle seule une question hautement politique, si l'on prend en considération que la majorité de la population mondiale est sous-alimentée. García met 
au centre de sa critique sévère l'univers McDonald's (RITZER, 1996, 1998, 2002), la société de l'exploitation économique mondialisée, la falsification culturelle, une consommation monstrueuse, autrement dit : les sociétés post-démocratiques.

\section{"À la bonne bouffe : aie pitié de l'Afrique et de l'Amérique latine » (GARCíA, 2011, 117)}

4 Ce qui caractérise la prise de position de l'auteur de la scène c'est l'histoire de Ronald, le clown de McDonald's (GARCíA, 2011, 33-65). L'association de l'alimentation et de la politique est évidente dès le titre, où l'on donne le nom de l'ancien président des ÉtatsUnis à l'immuable protagoniste des événements consommateurs de la chaîne McDonald's, celui qui nettoie les toilettes et distribue les célèbres happy meals aux petits enfants. La saleté de la première tâche contamine rapidement la seconde ainsi que le contenu même de ces plats. Le performer procède à une analyse détaillée de la composition des aliments.

Voici les succédanés de laitue, les succédanés de tomate et surtout ce morceau ovale et dur de couleur marron qui voudrait se faire passer pour un bout de vraie viande. À quoi il faut ajouter les liquides jaunes et rouges dont la formule n'a rien à voir ni avec la moutarde ni avec la sauce tomate.

Forcément, le mélange explosif de tous ces éléments placés dans cette petite boîte prodigieuse nommé Happy Meal provoque une grande quantité de gaz nocifs chez l'enfant qui l'ingère, avec une évidente répercussion éructo-cérébrale.

Celui qui mange ça, ne pourra plus jamais penser correctement de sa vie.

(Ibid., 2011, 52)

5 À côté de la maxime de la régularité médicale et nutritionniste : « Nous sommes ce que nous mangeons » (García dirait : « Nous sommes ce que nous ingérons » [COLLECTIF, 2005, 380]) il faut en ajouter une autre: "Nous pensons comme nous mangeons et en fonction de notre alimentation ». L'idéologie du repas bon marché et rapide, qui a dominé les décennies précédentes et qu'on a mise en avant dans le cadre d'une homogénéisation de la consommation alimentaire est ici totalement déconstruite, tout comme l'alibi démocratique d'une participation aux mêmes matériaux et produits culturels fonctionnant comme équilibrage pour la disparité sociale et la hiérarchie économique. C'est exactement sur la base de cette égalité abstraite des inégalités, sur celle de cette démocratie abstraite des fast foods que sont construits ces vrais systèmes de discriminations (BAUDRILLARD, 1989, 75-76).

Si tu as neuf ans et que tu vis à Lisbonne, tu vas au McDonald's le dimanche.

$\mathrm{Si}$ tu as neuf ans et que tu vis à Cuba, tu vas [...] d'un touriste italien.

Si tu as neuf ans et que tu vis à Bruxelles, tu vas au McDonald's le dimanche.

Si tu as neuf ans et que tu vis en Bolivie, tu vas à la mine pour les Américains.

Si tu as neuf ans et que tu vis à Florence, tu vas au McDonald's le dimanche.

Si tu as neuf ans et que tu vis en Afrique, tu couds des ballons pour Nike.

Si tu as neuf ans et que tu vis à New York, tu vas au McDonald's le dimanche.

Si tu as neuf ans et que tu vis en Thaillande, tu dois te laisser [...] par un Australien.

6 Tout comme la stratégie publicitaire, la propagande politique met toujours en avant la version qui profite aux plus forts, escamotant les faits et leurs paramètres structurels. Tout comme le discours publicitaire de Danone convainc de la pureté de ses produits, les puissants médias cherchent à convaincre les gens qu'un État a le droit de bombarder impunément Hiroshima ou de détruire l'Argentine sur le plan économique en invoquant le peuple blessé, le prestige blessé, l'ordre public blessé. 
Des troupes de soldats, des troupes de Donuts, des troupes de Big Macs, des troupes de Levis, des troupes de Philips, des troupes de Hollywood...

Quelle bande d'enculés!

(GARCÍA, 2011, 53)

7 Dès lors que, comme le souligne Baudrillard, on présuppose toujours l'existence d'un perpétuel excédent des besoins, les forces productives deviennent l'armée de réserve des besoins $(1989,75-76)$; c'est pour cette raison que García dirige aussi sa critique contre les consommateurs. Et le cercle vicieux se présente ainsi : nous mangeons les produits que nous contribuons à produire à travers notre comportement de consommateurs et ce que nous mangeons influe de façon destructrice sur notre mode de penser et sur son contenu (GARCíA, 2011, 50-51): non seulement cette pensée corrompue produite par des aliments corrompus ne peut résister à une consommation effrénée, mais elle la renforce. La pensée corrompue : la pensée altérée, "gâtée " (à l'instar d'une denrée alimentaire gâtée), la pensée «rance», pourrie, pensée dangereuse donc, pensée corrosive qui, quand elle entre en action (comme les denrées corrompues que l'on dévore) peut mener à la mort. «Plus les kilos de graisse sont nombreux, moins l'être a d'épaisseur" (COLlectif, 2005, 380). Ainsi, si à la fin du spectacle trois clowns de McDonald's font griller sur le barbecue des chefs-d'œuvre de la pensée universelle manifestement choisi dans le catalogue intitulé «En fouillant dans les ordures » (GARCíA, 2011, 64-65), où figurent entre autres Sophocle et Heidegger, Euripide et Levinas, Shakespeare et Aristote, ce n'est ni un hasard ni un simple choix fait pour éblouir. "Que se passe-t-il ensuite? Ces œuvres culturelles emblématiques sont consommées, arrosées de ketchup, entre deux pains à hamburger " (PAPALEXIoU, 2011). Mais il y a pire : on les mange ainsi après les avoir converties dans notre pensée en hamburgers, après les avoir corrompues, après les avoir tuées pour pouvoir les ingurgiter plus facilement.

8 Comme les vaccins qui injectent dans l'organisme le virus auquel ils s'opposent, la mort qui se donne à petites doses nous réconcilie progressivement avec l'obscurité finale. «Finalement chaque chose se nourrit de sa propre négation " (GARCíA, 2011, 385). Cela se réalise souvent à travers l'alimentation. La mort est à l'affût dans plusieurs textes, elle se cache derrière certains mots, elle attend l'apparition des plats, elle émerge de la puanteur de la pourriture ou de la métaphore du manger.

Dans Vous êtes tous des fils de pute « il y a des gens qui portent, toute leur vie, la mort en eux ». Après le Sein und Zeit de Heidegger (1986) ou les Essais hérétiques de Jan Patočka (1981), cette constatation n'est pas si saisissante puisque la mort, comme Derrida le souligne à ce propos, donne l'unicité, la singularité, l'irremplaçable, la responsabilité du moi $(1999,64)$, mais relie à nouveau la mort aux aliments: "Des gens Kinder Surprise/Tu les ouvres en deux et à l'intérieur/tout ce que tu trouves c'est des bricoles " (GARCÍA, 2011, 378). Le vide existentiel s'affiche ici affublé du masque de la mort et du vernis de la nourriture industrielle.

\section{"L'homme et la femme furent dotés des mêmes attributs que les chiens, les faisans, les thons et les hiboux : l'exaltation et l'émoi » (GARCíA, 2011, 53).}

C'est avec la projection de l'image de la cuisson de la viande sur le video wall que commence le spectacle intitulé "Bleue, saignante, à point, carbonisée »1. La société 
contemporaine occidentale en tant que hachoir à viande des pauvres. La société métaindustrielle de consommation est à l'homme - et qui plus est au plus pauvre et au plus faible - ce qu'est la boucherie aux animaux : l'espace où sa vie est dépecée, où il se trouvera réduit à un produit sans âme destiné à la consommation.

De ce point de vue, on pourrait expliquer pourquoi «l'animal est au fond notre hantise profonde, surtout l'animal qui est exposé pour ce qu'il est - une nourriture future, une chair à pâté qui vient nourrir toute l'économie humaine" (TACKELS, 2007, 61) - et pourquoi cette économie est intimement liée à la nourriture et à la mort. Il suffit d'écouter avec attention l'histoire de la vache devenue folle furieuse qui détruit tout autour d'elle quand elle sent qu'on lui a pris ses veaux pour les amener à l'abattoir (GARCíA, 2011, 46-49) ou de regarder les murgueros entrer en troupeaux comme les vaches, en une sinistre colonne d'animaux se dirigeant vers leur mise à mort. La consommation des animaux par l'homme fonctionne souvent comme une métaphore de la consommation de l'homme par l'homme. L'homme se nourrit d'animaux, comme il se nourrit de l'homme. Homo homini lupus. Nous nous nourrissons de la mort. C'est à sa façon - à travers le quotidien - que García aboutit à cette prise de conscience à laquelle les philosophes aboutissent par des chemins plus abstraits. C'est pour cela qu'il se focalise, de façon provocante, choquante même, sur les détails de la mise à mort de l'animal en tant que meurtre : la mise à mort est un acte officiel, ses origines remontent à très loin, alors que le meurtre prend ses racines dans l'ici et maintenant. C'est le cas de la représentation Accidens. Tuer pour manger (GARCíA, 2011, 157-159). Comme les futuristes et les dadaïstes du début $\mathrm{du} \mathrm{xx}^{\mathrm{e}}$ siècle, García cherche à provoquer des réactions fortes en choquant ses spectateurs quand il montre avec une clarté désarmante qu'un mets aussi cher que le homard, destiné bien sûr aux classes les plus aisées, cache le meurtre d'un animal. Le performer jette le homard dans l'eau brûlante ou le suspend à un fil hors de l'eau et l'arrose de temps à autre en l'empêchant de crever. Ensuite, devant les spectateurs et en commençant par ses pinces, il le découpe toujours vivant en morceaux puis le cuit et le mange, en fumant tranquillement un cigare et en sirotant du vin. Ce spectacle répugnant touchera à l'hideux quand des amplificateurs spéciaux achemineront dans la salle du théâtre les sonorités puissantes du cœur du crustacé approchant de la mort ou au moment du dépeçage ou de son " exécution ». Bruno Tackels met ce dernier mot entre guillemets, puisque cette " mise à mort est mise en scène » et il se demande pourquoi la mise à mort du homard "ne nous touche [...] que lorsqu'elle est visible » et pourquoi nous n'en parlons pas en dehors de la scène, dans un bon restaurant par exemple. Son hypothèse est que la scène retient toujours la dimension sacrée et que par conséquent elle ritualise et sacralise le profane et le quotidien $(2007,61-62)$. Je ne partage pas son point de vue. Il est vrai, comme le souligne Stourna, que cette performance est conçue à la limite du représentable et de l'irreprésentable et que la mise à mort du homard sur la scène peut être considérée comme outrancière $(2011,176)$. Mais aucun cadre théâtral, même celui qui invoque sa dimension rituelle ne peut sacraliser la perpétration d'un meurtre (PEFANIS, 2007, 159-193). Il y a quelque chose dans le homard qui tient de l'homme et quelque chose dans l'homme contemporain qui rappelle la destination du homard, dans la mesure où tous les deux seront victimes d'un système qui engloutit aussi facilement les chairs que les désirs. 


\section{« Ton corps voyage et toi, tu portes la mort dans ton corps »}

Dans Golgota picnic (GARCíA, 2011a)², l'acte de manger et le fantôme de la mort tiennent une place de premier ordre. Ces deux sujets jettent de mille façons leur ombre sur le texte comme sur la représentation de l'œuvre. Le mortel et le mangeable, la mort et le manger dans cette œuvre créent un contraste intéressant. Voyons d'abord les images scéniques. Le performer-Christ est "mis en croix » sur le sol envahi de petits pains pour hamburgers. Les visages sont recouverts de légumes. Le hachoir à viande fait ici aussi son apparition et juste après, un homme nu allongé sur le ventre sur les petits pains, le visage complètement recouvert de viande hachée. S'agit-il d'un visage écrasé, de cervelles répandues, de pensées qui se sont déversées hors de la tête en une sorte de brainstream cauchemardesque, de pensées qui ne sont rien d'autre que de la viande hachée ou de la mort incarnée scéniquement par la viande ? Un hamburger géant se construit peu à peu, mais au lieu de viande « morte » des abattoirs, ici c'est de la viande « vivante » qui fait son apparition, c'est-à-dire des vers grouillant au milieu des petits pains entassés et qui le détruisent comme sont détruits les splendides constructions de la culture occidentale, les règles alimentaires et la vie contemporaine elle-même. Ce qui nourrit la vie contemporaine est ce qui la mène à la mort : la nourriture-mort. Sartre écrit dans l'Être et le Néant : « Manger, en effet, c'est s'approprier par destruction, c'est en même temps se boucher avec un certain être » (SARTRE, 1986, 676).

\section{Paysages anthropophagiques de Vassilis Ziogas}

Dans Restaurant « Humanismus» de Vassilis Ziogas (ZIOGAS, 1996, 9-27)3 , l'acte de manger ne peut être dissocié du mourir. Plus précisément, ici le mortel correspond au mangeable. Si García utilise des images de boucherie, Ziogas estime que le rôle de l'homme décadent est d'être mangé pendu dans une boucherie. Les restaurants servent de la nourriture de seconde qualité, c'est-à-dire des hommes d'Amérique latine ou des miséreux venant de la rue. Certains proposent une viande de chimpanzé pour de la viande humaine (ZIOGAS, 1996, 21). L'amour passe littéralement par le ventre puisqu'une nourriture savoureuse et saine ne peut se trouver que dans le corps de la femme.

L'anthropophagie est une expression ontologique puisqu'elle étend le mortel (ßpotó) par le biais du mangeable ( $\beta \rho \omega \tau$ tós) et qu'elle met en évidence que le corps humain est simultanément sujet et objet de l'acte de manger ( $\beta \rho \omega ́ \sigma \eta)$, consommant et consommé, qu'il mange et il est mangé. Les personnages de Ziogas, dans une scène que caractérisent l'opulence, le luxe et l'affectation, se nourrissent de la mort, puisqu'ils mangent les hommes qui ont été tués un peu plus tôt justement pour être mangés ; au même instant cependant ils nourrissent la mort, puisque tant qu'ils se nourrissent de ces mets rares et coûteux ils ne font pas que confirmer les meurtres d'humains, ils ne font pas que renforcer le rituel anthropophage, ils endossent en plus une dette difficilement remboursable: pour avoir contracté une dette insoutenable ils deviennent donc débiteurs, ce qui mine peu à peu leur propre existence.

Le Restaurant « Humanismus » est une dystopia satirique qui éclaire ce dont la langue ellemême témoigne en chuchotant, c'est-à-dire que l'acte de manger n'est rien d'autre que la nourriture des mortels, l'énergie et le contenu de cette énergie qui nourrissent le mortel en tant que mortel. Cette phrase a trois significations qui se ressemblent, mais ne peuvent pourtant pas cacher les tensions qui existent entre elles. Tout d'abord la 
signification commune selon laquelle l'acte de manger donne vie au mortel, facilite sa survie en satisfaisant ses besoins alimentaires: je me nourris, donc «je continue à vivre » et au « continue » fait doucement écho le « mon dernier jour est différé ». Même le simple fait de se sustenter ne peut adoucir une trace de mort même infime, à plus forte raison, dès lors que tout ce qui entre dans l'organisme humain est apte à le conserver ou à le détruire. Bien sûr, la mort se cache aussi ailleurs: avec le "nourrissent ", discrètement mais sûrement, on présuppose déjà la mort de celui qui devient nourriture pour l'homme (qu'il soit plante ou animal).

Vient ensuite la signification où l'acte de manger conserve le mortel en tant que tel, en tant que mortel, en tant que être-pour-la-mort. Ici, le $\beta i \beta \rho \omega \sigma \kappa \omega$ (dévorer) renvoie au $\delta i \alpha \beta \imath \rho \omega \sigma \sigma \kappa \omega$ (corroder), la $\beta \rho \omega ́ \sigma \eta$ (l'acte de manger) et la $\delta i \alpha ́ \beta \rho \omega \sigma \eta$ (corrosion) s'entrelacent, par le biais de ce « $\delta i \alpha »$ ajouté qui renvoie à une forme réflexive, le contenu de l'acte de manger ( $\beta \rho \omega ́ \sigma \eta)$ n'étant pas l'ambroisie (les dieux immortels se

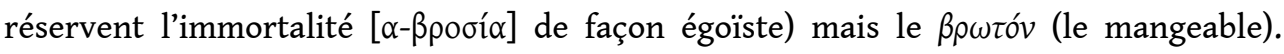

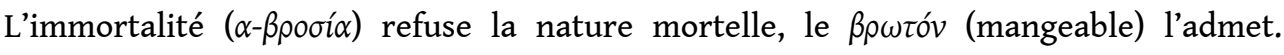
Autrement dit : la nourriture affirme la mort.

C'est finalement avec une troisième signification que j'achèverai ces réflexions. Tournons notre attention vers la notion d'incorporation pour qu'apparaisse le travail du deuil, comme travail du survivre. Arrêtons-nous sur les mots de la lettre du comte Dendé que « la mort est une chaîne de vie » (ZIOGAs, 1996, 25). C'est-à-dire que ce qu'on récapitule par le mot "mort ", c'est une chaîne de vies perdues, mais perdues pour qui ? Les vies perdues qui nourrissent la mort présupposent toujours l'existence de celui auquel elles manquent et pour lequel elles sont perdues. La vie perdue est perdue parce qu'elle a un témoin qui témoigne de la perdition et pour qui la perdition est une perte ; un témoin qui incorpore la perte dans son existence, qui porte le deuil du disparu, qui survit à sa mort, en une survivance qui se constitue grâce à cette mort (DERRIDA, 1986, 117-218). La survivance n'est pas une simple survie : elle n'est pas une postériorité comparative pendant laquelle la vie de l'un continue après la mort de l'autre, mais c'est la vie qui se nourrit de la mort de l'autre, qui incorpore sa perte mettant en suspension la conscience qui la conçoit, puisque c'est la conscience d'un survivant en suspension (DERRIDA, 2006, 27).

Par conséquent, la phrase du comte Dendé «la mort est une chaîne de vie » porte en son sein son inverse : « la vie est une chaîne de mort ", une chaîne de survivances, une suite d'entailles où les morts laissent leurs traces. Les traces pour Ziogas, s'inscrivent dans le corps des survivants. Voyons la suite de la lettre : «Et la meilleure tombe est le corps d'un ami très cher. Je te salue du fond de ton estomac ». La mort du défunt nourrit le corps du survivant et Ziogas illustre littéralement ce "nourrit ». Les vies perdues nous apprennent, comme Derrida l'a justement écrit, à vivre enfin $(1993,13) \grave{a}$ vivre au bout de la vie, en nous nourrissant de mort. 


\section{BIBLIOGRAPHIE}

BAUDRILLARD Jean, 1989, la Société de consommation, Paris : Gallimard.

COLLECTIF, 2005, Mises en scène du monde, Besançon : Les solitaires intempestifs.

DERRIDA Jacques, 1986, Parages, Paris : Galilée.

DERRIDA Jacques, 1993, Spectres de Marx : l'État de la dette, le travail du deuil et la nouvelle

Internationale, Paris : Galilée.

DERRIDA Jacques, 1999, Donner la mort, Paris : Galilée.

DERRIDA Jacques, 2006, Apprendre à vivre enfin, Entretien avec Jean Birnbaum, (en grec), Athènes :

Agra.

FISCHLER Claude, 2001, l'Homnivore: le goût, la cuisine et le corps, Paris : Odile Jacob.

GARCíA Rodrigo, 2009, Bleue, saignante, à point, carbonisée, Besançon : Les solitaires intempestifs.

GARCíA Rodrigo, 2011, Cendres 2000-2009, Besançon : Les solitaires intempestifs.

GARCÍA Rodrigo, 2011a, Golgota picnic, Besançon : Les solitaires intempestifs.

HEIDEGGER Martin, 1986, Etre et temps, Paris : Gallimard.

PAPALEXIOU Eleni, 2011, « Le théâtre politique de Rodrigo García », in http://www.academia.edu/ 698153/, Centre de recherche sur l'histoire du théâtre, Université Paris IV-Sorbonne.

PATOČKA Jan, 1981, Essais hérétiques sur la philosophie de l'histoire, [1 ${ }^{\text {re }}$ éd., Prague, 1975], Paris :

Verdier.

PEFANIS Georges P., 2007, Scènes de la théorie: des champs de la théorie et la critique du théâtre, Athènes : Papazisis.

POULAIN Jean-Pierre, 2013, Sociologies de l'alimentation : les mangeurs et l'espace social alimentaire, Paris : PUF.

RITZER George, 1996, The Mcdonaldization of Society, Thousand Oaks, CA, Pine Forge Press,

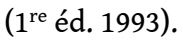

RITZER George, 1998, The Mcdonaldization Thesis, Explorations and Extentions, London: Sage.

RITZER George (ed.), 2002, Mcdonaldization: The Reader, Thousand Oaks CA Pine Forge Press, and London: Sage.

SARTRE Jean-Paul, 1986, l'Être et le Néant : essai d'ontologie phénoménologique, Paris : Gallimard.

STOURNA Athéna-Hélène, 2011, la Cuisine à la scène : boire et manger au théâtre du XX ${ }^{e}$ siècle, Rennes Tours : Presses Universitaires de Rennes-Presses Universitaires François Rabelais de Tours.

TACKELS Bruno, 2007, Rodrigo García : écrivains de plateau IV, Besançon : Les solitaires intempestifs.

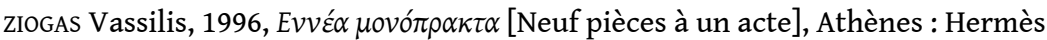




\section{NOTES}

1. Le spectacle a été créé le 14 juin 2007 au ${ }^{\mathrm{e}}{ }^{\mathrm{e}}$ Festival international des Arts de Castille et León à l'Espace Fonseca de Salamanque et le 20 juin 2007 au Festival d'Athènes et d'Épidaure dans une mise en scène de l'auteur.

2. La pièce a été présentée le 7 janvier 2011 au Théâtre María Querrero (Centre Dramatique National de Madrid) dans une mise en scène de l'auteur. Elle a ensuite été jouée en France au Théâtre Garonne de Toulouse le 16 novembre 2011 puis dans le cadre du Festival d'Automne à Paris. En Grèce elle a été jouée en 2012 au Festival d'Athènes et d'Épidaure.

3. La pièce a été écrite à Paris en 1958 et elle a été jouée pour la première fois en 1963 à Athènes par le Théâtre Expérimental de Poche de Marietta Rialdi dans une mise en scène de Minas Cristidis.

\section{RÉSUMÉS}

Cet essai rapproche certaines pièces de Rodrigo García et Vassilis Ziogas, afin d'examiner la possibilité d'un passage caché entre $\beta \rho \omega ́ \sigma \eta \varsigma$ (manger) et $\delta i \alpha ́ \beta \rho \omega \sigma \eta \varsigma$ (éroder), d'une liaison latente

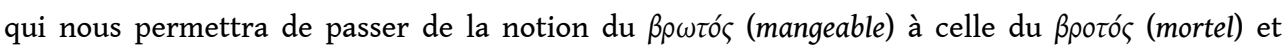
associer le repas au mort sur la scène.

One of the most important functions of human life, eating food is also a very frequent and meaningful scene in contemporary theatre productions. In these scenes and in the frame of the consumerist culture of late capitalism, eating food represent sometimes voraciousness and gluttony in a contrast with ultimate hunger or refer to "haute cuisine" and cannibalism. Rodrigo García's Golgota picnic (Théâtre du Rond Point, Paris, 2011 and Athens Festival, 2012) and Bleue, saignante, à point, carbonisée (Avignon and Athens Festival, 2007), as well as Vassilis Ziogas's Restaurant "Humanismus" (Paris 1958) are three of the plays that will be examined in this paper.

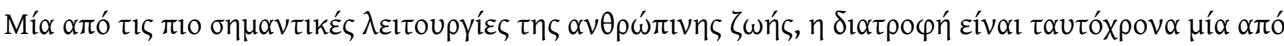

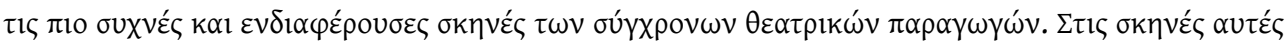

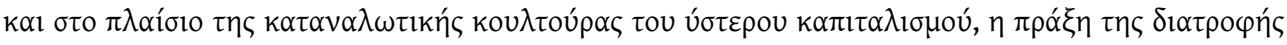

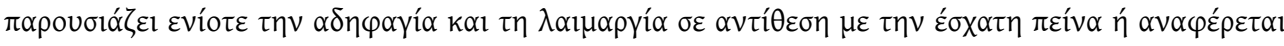

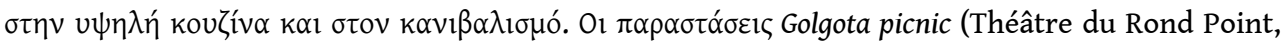

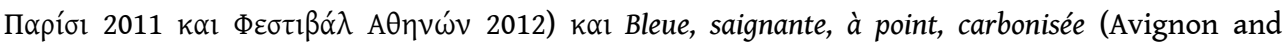

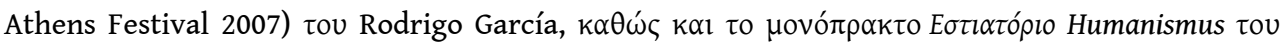

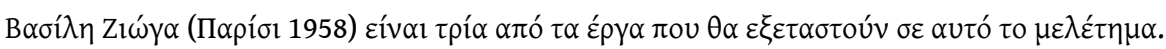


INDEX

Index géographique : Grèce

motsclesmk СНАБДУВАњЕ, ВГРАДУВАҢЕ, КАНИБАЛИЗА М, М Р СНАБДУ ВАЮЕ, КАНИБАЛИЗАМ, МРТОВ, КАПИТАЛИЗАМ, ПРЕЖИВУВАњЕ, ГРЦИЈА, ДВАЕСЕТТИОТ ВЕК, ТЕАТАР

Keywords : Alimentation, Incorporation, Cannibalism, Death, Capitalism, Survival, Greece, Twentieth Century, Theatre

motsclestr Arz, Birleşme, Yamyamlık, Ölüm, Kapitalizm, Hayatta kalma, Yunanistan, Yirminci yüzyıl, Tiyatro

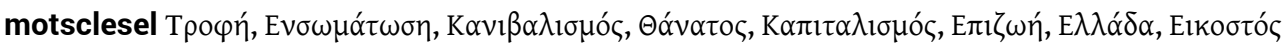
$\alpha \imath \omega ́ v \alpha \varsigma, \Theta \varepsilon ́ \alpha \tau \rho o$

Mots-clés : alimentation, incorporation, incorporation, cannibalisme, cannibalisme, mort, mort, capitalisme, capitalisme, survie, survie

Thèmes : Théâtre

Index chronologique : vingtième siècle

\section{AUTEUR}

\section{GEORGES P. PEFANIS}

Université Nationale et Capodistrienne d'Athènes 